\title{
The Center of a Convex Set and Capital Allocation
}

\author{
Bogdan Grechuk \\ Department of Mathematics, University of Leicester, LE1 7RH, UK \\ bg83@leicester.ac.uk
}

\begin{abstract}
A capital allocation scheme for a company that has a random total profit $Y$ and uses a coherent risk measure $\rho$ has been suggested. The scheme returns a unique real number $\Lambda_{\rho}^{*}(X, Y)$, which determines the capital that should be allocated to company's subsidiary with random profit $X$. The resulting capital allocation is linear and diversifying as defined by Kalkbrener (2005). The problem is reduced to selecting the "center" of a non-empty convex weakly compact subset of a Banach space, and the solution to the latter problem proposed by Lim (1981) has been used. Our scheme can also be applied to selecting the unique Pareto optimal allocation in a wide class of optimal risk sharing problems.
\end{abstract}

Key Words: capital allocation, risk contribution, coherent risk measures, risk sharing.

\section{Introduction}

One of the basic problems in risk management is to determine the allocation of risk capital among agents or business units. We make two assumptions: (i) a company consists of $n$ subsidiaries, each contributing a random profit $X_{i}$, so that the total profit of the company is $Y^{*}=\sum_{i=1}^{n} X_{i}$, and (ii) the company has decided, or is required by a regulator, to reserve a risk capital $\rho\left(Y^{*}\right)$, to compensate possible loss, where $\rho($.$) is a$ fixed risk measure. The capital allocation problem is to distribute $\rho\left(Y^{*}\right)$ among subsidiaries, that is, to assign subsidiary $i$ the capital $k_{i}$ with $\sum_{i=1}^{n} k_{i}=\rho\left(Y^{*}\right)$. The numbers $k_{i}$ are called risk contributions of $X_{i}$ to the $Y^{*}$.

If $\rho\left(Y^{*}\right) \leq \sum_{i=1}^{n} \rho\left(X_{i}\right)$, it is possible to find a capital allocation such that $k_{i} \leq \rho\left(X_{i}\right), i=1, \ldots, n$. This corresponds to the intuition of diversification: the risks produced by subsidiaries partially compensate each other, which allows one to reduce the risk contribution of each of them. If $\rho\left(Y^{*}\right)=\sum_{i=1}^{n} \rho\left(X_{i}\right)$, such an allocation is unique and given by $k_{i}=\rho\left(X_{i}\right), i=1, \ldots, n$. The capital allocation problem can be formulated as follows.

Problem I Assume that $\rho\left(Y^{*}\right)<\sum_{i=1}^{n} \rho\left(X_{i}\right)$, so that a capital allocation satisfying $k_{i} \leq \rho\left(X_{i}\right), i=1, \ldots, n$ is not unique. Which one to choose?

The capital allocation problem in this or similar form has been extensively studied in a number of papers, see eg. Denault (2001), Delbaen (2004), Kalkbrener (2005), Cherny and Orlov (2011) and references therein. We rely on a natural assumption (see Kalkbrener (2005)), that the risk contribution $k_{i}$ of subsidiary $i$ depends only on $X_{i}$ and $Y^{*}$, but not on the decomposition of $Y^{*}-X_{i}$ among the rest of subsidiaries. In this context, a capital allocation with respect to risk measure $\rho($.$) is just a function of two ar-$ guments $\Lambda_{\rho}(X, Y)$, such that $\Lambda_{\rho}(Y, Y)=\rho(Y)$. With $k_{i}=\Lambda_{\rho}\left(X_{i}, Y^{*}\right)$, the requirements (i) $\sum_{i=1}^{n} k_{i}=\rho\left(Y^{*}\right)$ and (ii) $k_{i} \leq \rho\left(X_{i}\right), i=1, \ldots, n$ can now be rewritten as

(i) (Linearity) $\Lambda_{\rho}(X, Y)$ is a linear functional in the first argument;

(ii) (Diversification) $\Lambda_{\rho}(X, Y) \leq \rho(X)$ for all $X, Y$.

Kalkbrener (2005) proved that a linear diversifying capital allocation exists if and only if the risk measure $\rho$ is positively homogeneous and subadditive. He also proved that it is unique for a fixed $Y$ and $\rho($.$) if and$ only if the directional derivative

$$
\lim _{\varepsilon \rightarrow 0} \frac{\rho(Y+\varepsilon X)-\rho(Y)}{\varepsilon}
$$

exists for all $X$. This, however, is not the case in many natural contexts. Cherny and Orlov (2011) argue that it might not exists for a popular risk measure $C \operatorname{VaR}($.$) if$ the distribution function of $Y$ is not continuous, which 
is typical e.g. in the context of credit portfolios. Actually, if we denote $\mathcal{Y}(\rho)$ to be the set of all $Y$ such that (1) does not exists for some $X$, one can prove that $\mathcal{Y}(\rho)$ is non-empty unless $\rho($.$) is linear (see Propo-$ sition 1), and, moreover, one may get $Y \in \mathcal{Y}(\rho)$ by solving a natural optimization problem (see Example 3 ). Problem I can be adapted to this context as follows

Problem II Let $\rho$ be a positively homogeneous and subadditive risk measure, and assume that $Y \in$ $\mathcal{Y}(\rho)$, so that a linear diversifying capital allocation $\Lambda_{\rho}(X, Y)$ is not unique. Which one to choose?

A possible approach to solve Problem II is to impose additional assumptions on capital allocation. Kalkbrener (2005) introduced a "continuity in $Y$ " axiom, and proved that if a linear, diversifying, and continuous capital allocation exists, it is unique. However, Theorem 4.3. in Kalkbrener (2005) states that the existence of such a capital allocation is equivalent to the existence of directional derivative in (1), so this approach makes no progress in Problem II.

Cherny and Orlov (2011) suggested to consider the capital allocation problem within the framework of coherent risk measures of Artzner et al. (1999), and introduced an assumption that $\Lambda_{\rho}(X, Y)$ is law-invariant, that is, depends only on the joint law of $X$ and $Y$. They proved that the linear diversifying law-invariant capital allocation exists and is unique for a class of so called weighted $V a R$ risk measures, defined on an atomless probability space. However, there are important coherent risk measures outside of this class, for which such a capital allocation does not exist or might not be unique, see Section 2.

In this paper, we develop an approach of selecting one linear diversifying capital allocation for every coherent risk measure. Our approach can naturally be extended to other classes of positively homogeneous and subadditive functionals. We do this by reducing this problem to selecting a "center" in a convex weakly compact subset of a Banach space, which is fixed under affine isometries, and the latter problem was solved in Lim (1981). If directional derivative in (1) exists for every $X$, our capital allocation coincides with that of Kalkbrener (2005); if $\rho$ belongs to the class of weighted $\mathrm{VaR}$, it coincides with that of Cherny and Orlov (2011). We do not assume that probability space is atomless, and an important special case of discrete probability space is treated separately.
This work is organized into eight sections. Section 2 formulates a capital allocation problem, provides several motivating examples, and reduces the problem to identification of the "center" in a convex weakly compact set. Section 3 suggests a solution in a special but important case when the underlying probability space is finite. Section 4 reformulates the concept of lawinvariant capital allocation, suggested by Cherny and Orlov (2011), in terms of invariance under automorphisms of the underlying probability space. Section 5 solves the capital allocation problem in the general case, while Section 6 discusses an economic interpretation of the obtained solution. Section 7 applies the results to the optimal risk sharing problem. Section 8 concludes the work.

\section{Problem Formulation}

Let $(\Omega, \mathcal{F}, \mathbb{P})$ be a probability space, where $\Omega$ denotes the designated space of future states $\omega, \mathcal{F}$ is a field of sets in $\Omega$, and $\mathbb{P}$ is a probability measure on $(\Omega, \mathcal{F})$. We will assume that probability space is standard, that is, isomorphic mod $0^{1}$ to an interval $(0,1)$ with Lebesgue measure, a finite or countable set of atoms ${ }^{2}$, or a combination (disjoint union) of both. A random variable (r.v.) is any measurable function from $\Omega$ to $\mathbb{R}$. The relations between r.v.'s are understood to hold in the almost sure sense, e.g., we write $X=Y$ if $\mathbb{P}[X=Y]=1$ and $X \geq Y$ if $\mathbb{P}[X \geq Y]=1$. We restrict our attention to r.v.'s from $\mathcal{L}^{1}(\Omega)=\mathcal{L}^{1}(\Omega, \mathcal{F}, \mathbb{P})$ (r.v.s with finite expectations), or from $\mathcal{L}^{\infty}(\Omega)=\mathcal{L}^{\infty}(\Omega, \mathcal{F}, \mathbb{P})$ (bounded r.v.s). $F_{X}(x)=\mathbb{P}[X \leq x]$ and $q_{X}(\alpha)=\inf \left\{x \mid F_{X}(x)>\alpha\right\}$ will denote the cumulative distribution function (CDF) and quantile function of an r.v. $X$, respectively. The probability space $\Omega$ is called atomless if there exists an r.v. with a continuous CDF. This implies existence of r.v.'s on $\Omega$ with all possible CDFs (see e.g. Proposition A.27 in Föllmer and Schied (2004)).

A risk measure is any functional $\rho: \mathcal{L}^{\infty}(\Omega) \rightarrow \mathbb{R}$. A capital allocation with respect to the risk mea-

\footnotetext{
${ }^{1}$ Two probability spaces $\left(\Omega_{1}, \mathcal{F}_{1}, \mathbb{P}_{1}\right)$ and $\left(\Omega_{2}, \mathcal{F}_{2}, \mathbb{P}_{2}\right)$, are isomorphic $\bmod 0$, if there exist sets $A_{i} \subset \Omega_{i}, i=1,2$, with $\mathbb{P}_{1}\left(A_{1}\right)=$ $\mathbb{P}_{2}\left(A_{2}\right)=0$, and an invertible map $f: \Omega_{1} / A_{1} \rightarrow \Omega_{2} / A_{2}$ such that both $f$ and $f^{-1}$ are measurable, and $\mathbb{P}_{1}(A)=\mathbb{P}_{2}(f(A))$ for all $A \in \mathcal{F}_{1}$.

${ }^{2}$ A set $A \in \mathcal{F}$ with $\mathbb{P}[A]>0$ is called atom if $\mathbb{P}[B]=0$ or $\mathbb{P}[B]=$ $\mathbb{P}[A]$ for every $B \in \mathcal{F}$ such that $B \subset A$.
} 
sure $\rho($.$) is a functional \Lambda_{\rho}: \mathcal{L}^{\infty}(\Omega) \times \mathcal{L}^{\infty}(\Omega) \rightarrow \mathbb{R}$ satisfying $\Lambda_{\rho}(Y, Y)=\rho(Y)$. It is called linear if $\Lambda_{\rho}\left(a X_{1}+b X_{2}, Y\right)=a \Lambda_{\rho}\left(X_{1}, Y\right)+b \Lambda_{\rho}\left(X_{2}, Y\right)$, for all $X_{1}, X_{2}, Y \in \mathcal{L}^{\infty}(\Omega)$ and $a, b \in \mathbb{R}$; it is called diversifying if $\Lambda_{\rho}(X, Y) \leq \rho(X)$ for all $X, Y \in \mathcal{L}^{\infty}(\Omega)$. This paper studies the problem of selection of a linear diversifying capital allocation for a given risk measure $\rho($.$) .$

Theorem 4.2. in Kalkbrener (2005) proves that there exists a linear diversifying capital allocation $\Lambda_{\rho}(.,$.$) if$ and only if $\rho($.$) is positively homogeneous and subad-$ ditive. This suggests that the capital allocation problem can naturally be considered in the context of $\mathrm{co}$ herent risk measures in the sense of Artzner et al. (1999).

Definition 1 (coherent risk measures). A coherent risk measure is any functional $\rho: \mathcal{L}^{\infty}(\Omega) \rightarrow \mathbb{R}$ satisfying the axioms

(Rl) $\rho(X) \leq \rho(Y)$ if $X \geq Y$ (monotonicity),

(R2) $\rho(\lambda X)=\lambda \rho(X)$ for all $X$ and all $\lambda \geq 0$ (positive homogeneity),

(R3) $\rho(X+Y) \leq \rho(X)+\rho(Y)$ for all $X$ and $Y$ (subadditivity),

(R4) $\rho(X+C)=\rho(X)-C$ for all $X$ and constant $C$.

A risk measure $\rho($.$) is called continuous from be-$ low if $\lim _{n \rightarrow \infty} \rho\left(Y_{n}\right)=\rho(Y)$ for every non-decreasing sequence $Y_{n}$ converging to $Y$ pointwise on $\Omega$. For simplicity of presentation, we first solve the capital allocation problem for the case of coherent risk measures, which are continuous from below. The extension to all coherent risk measures, and beyond, is presented in Section 5.

Corollary 4.35 in Föllmer and Shield (2004) states that coherent risk measure $\rho$ is continuous from below if and only if

$$
\rho(Y)=\max _{Q \in Q} E_{Q}[-Y], \text { for all } Y \in \mathcal{L}^{\infty}(\Omega),
$$

for $Q \subset \mathcal{F}_{1}(\mathbb{P})$, where $\mathcal{F}_{1}(\mathbb{P})$ is the set of all probability measures which are absolutely continuous with respect to $\mathbb{P}$. Moreover, $Q$ can be chosen such that the set of Radon-Nikodym derivatives

$$
\mathcal{S}=\left\{\frac{d Q}{d \mathbb{P}}, Q \in Q\right\}
$$

is weakly compact in $\mathcal{L}^{1}(\Omega)$. Also, without loss in generality, $Q$ (and thus $\mathcal{S}$ ) can be assumed to be convex, otherwise the same representation holds for its closed convex hull. For a fixed $Y \in \mathcal{L}^{\infty}(\Omega)$, let $\mathcal{S}(Y)$ be the non-empty set of elements of $\mathcal{S}$, at which the maximum in (2) is attained. For every $S=\frac{d Q}{d \mathbb{P}} \in S(Y)$, the functional $\Lambda_{\rho}(X, Y):=E_{Q}[-X]=-E[S X]$ is a linear diversifying capital allocation. Theorem 4.3. in Kalkbrener (2005) states that it is unique (and thus $S(Y)$ is a singleton) if and only if the directional derivative (1) exists for all $X$. For a given risk measure $\rho$, let $\mathcal{Y}(\rho)$ be the set of all $Y$ such that $S(Y)$ is not a singleton (equivalently, such that (1) does not exist for some $X$ ). We start with the following simple observation.

Proposition 1 For risk measure $\rho$ satisfying $(R 2)$ and $(R 3)$, the directional derivative (1) exists at 0 in any direction $X$, if and only if $\rho$ is linear. Hence $0 \in \mathcal{Y}(\rho)$ if and only if $\rho$ is not linear.

Proof If $\rho$ is linear, the directional derivative (1) exists and equal to $\rho(X)$. In the opposite direction, if the derivative (1) exists for $Y=0$, then the right derivative, which is $\rho(X)$, is equal to the left one, which is $-\rho(-X)$. Hence, using subadditivity, $\rho\left(X_{1}+X_{2}\right) \leq$ $\rho\left(X_{1}\right)+\rho\left(X_{2}\right)=-\left(\rho\left(-X_{1}\right)+\rho\left(-X_{2}\right)\right) \leq-\rho\left(-X_{1}-\right.$ $\left.X_{2}\right)=\rho\left(X_{1}+X_{2}\right)$, showing $\rho\left(X_{1}+X_{2}\right)=\rho\left(X_{1}\right)+$ $\rho\left(X_{2}\right)$ for any $X_{1}, X_{2} \in \mathcal{L}^{\infty}(\Omega)$.

Proposition 1 shows that, for every non-linear $\rho$, set $\mathcal{Y}(\rho)$ contains 0 , and therefore, in particular, it is nonempty. One may argue that the set $\mathcal{Y}(\rho)$ is typically non-empty but the event $Y \in \mathcal{Y}(\rho)$ is rather exceptional. This is supported by Corollary 5.1. in Kalkbrener (2005), which states that $\mathcal{Y}(\rho)$ consists only on constants for risk measure $\rho_{S T D}(X)=c \cdot \sigma(X)-E X$, where $\sigma(X)=\|X-E X\|_{2}$ is the standard deviation. However, $\rho_{S T D}$ is not a coherent risk measure (axiom $\mathrm{R} 1$ fails), and, in applications, is sometimes replaced by $\rho_{M A D}(X)=c\|X-E X\|_{1}-E X$, which is coherent for $c \in\left[0, \frac{1}{2}\right]$, see Rockafellar et al. (2006a).

Example 1 For a coherent risk measure $\rho_{M A D}(Y)=$ $c\|Y-E Y\|_{1}-E Y, \quad c \in\left(0, \frac{1}{2}\right]$, we have $\mathcal{Y}(\rho)=$ $\{Y: P(Y=E Y)>0\}$.

Detail. For $\rho_{M A D}$, the set $\mathcal{S}$ in (3) is given by $S=\{S: E S=1, \sup S-\inf S \leq 2 c\}$, and, for nonconstant $Y, S(Y)=1+c(E Z-Z), Z \in \operatorname{sign}[Y-E Y]$, 
where $\operatorname{sign}[X]$ is a set of r.v.s $Z$ such that $Z(\omega)=1$, $Z(\omega)=-1$, and $Z(\omega) \in[-1,1]$ for $X(\omega)>0, X(\omega)<$ 0 , and $X(\omega)=0$, correspondingly, see Rockafellar et al. (2006b). If $Y$ is a constant, $S(Y)=S$. Thus, in any case, $\mathcal{S}(Y)$ is not a singleton if and only if $P(Y=E Y)>0$.

In the above example, the event $\{Y \in \mathcal{Y}(\rho)\}=$ $\{P(Y=E Y)>0\}$ may still be considered as almost negligible for practice. The next examples demonstrate that this is not the case for some other important risk measures.

Example 2 For a coherent risk measure given by

$\rho(Y)=\max _{i \in\{1,2\}} E\left[Q_{i}(-Y)\right], Q_{1}, Q_{2} \in \mathcal{F}_{1}(P), Q_{1} \neq Q_{2}$,

we have $\mathcal{Y}(\rho)=\left\{Y: E_{Q_{1}}[-Y]=E_{Q_{2}}[-Y]\right\}$.

Detail. Let $Y$ be such that $E_{Q_{1}}[-Y]=E_{Q_{2}}[-Y]$. Because $Q_{1} \neq Q_{2}$, we have $Q_{1}(A)<Q_{2}(A)$ for some set $A \in \mathcal{F}$, and $E_{Q_{1}}[-X]>E_{Q_{2}}[-X]$ for the indicator function $X=I_{A}$ of this set. Then $\rho(Y+\varepsilon X)=E_{Q_{1}}[-(Y+$ $\varepsilon X)]$ for $\varepsilon>0$, and $\rho(Y+\varepsilon X)=E_{Q_{2}}[-(Y+\varepsilon X)]$ for $\varepsilon<0$, whence the right and left directional derivatives in (1) are $E_{Q_{1}}[-X]$ and $E_{Q_{2}}[-X]$, correspondingly.

The risk measure (4) has the following interpretation: a company may consider two possible probabilistic scenarios with Radon-Nikodym derivatives $Q_{1}$ and $Q_{2}$, and the risk of every random profit $Y$ is just the worst-case expected loss. Now, assume that the company can choose a policy from a set of admissible policies, leading to a set of possible profits $\mathcal{Y} \subset \mathcal{L}^{\infty}(\Omega)$, that is, to the optimization problem

$$
\inf _{Y \in \mathcal{Y}} \rho(Y) .
$$

We will call problem (5) reducible, if an optimizer in (5) solves also optimization problem $\inf _{Y \in \mathcal{Y}} E_{Q_{1}}[-Y]$ or $\inf _{Y \in \mathcal{Y}} E_{Q_{2}}[-Y]$ (that is, one of the scenarios can be neglected), and irreducible otherwise.

Example 3 Let $\rho$ be given by (4), and let $\mathcal{Y} \subset \mathcal{L}^{\infty}(\Omega)$ be a convex set such that problem (5) is irreducible. Then any minimizer $Y^{*}$ in problem (5), if it exists, belongs to $\mathcal{Y}(\rho)$.

Detail. Because problem (5) is irreducible, set $\mathcal{Y}$ contains r.v.s $Y_{1}, Y_{2}$ such that $E_{Q_{i}}\left[-Y_{i}\right]<E_{Q_{i}}\left[-Y^{*}\right], i=$
1,2. For every $\lambda \in(0,1), \lambda Y^{*}+(1-\lambda) Y_{1} \in \mathcal{Y}$, whence

$$
\begin{aligned}
E_{Q_{1}}\left[-Y^{*}\right] & \leq \rho\left(Y^{*}\right) \leq \rho\left(\lambda Y^{*}+(1-\lambda) Y_{1}\right) \\
& =\max _{i \in\{1,2\}} \lambda E_{Q_{i}}\left[-Y^{*}\right]+(1-\lambda) E_{Q_{i}}\left[-Y_{1}\right] .
\end{aligned}
$$

But $E_{Q_{1}}\left[-Y^{*}\right]=\lambda E_{Q_{1}}\left[-Y^{*}\right]+(1-\lambda) E_{Q_{1}}\left[-Y^{*}\right]>$ $\lambda E_{Q_{1}}\left[-Y^{*}\right]+(1-\lambda) E_{Q_{1}}\left[-Y_{1}\right]$, whence

$E_{Q_{1}}\left[-Y^{*}\right] \leq \lambda E_{Q_{2}}\left[-Y^{*}\right]+(1-\lambda) E_{Q_{2}}\left[-Y_{1}\right], \forall \lambda \in(0,1)$.

Taking $\lambda \rightarrow 1$, we get $E_{Q_{1}}\left[-Y^{*}\right] \leq E_{Q_{2}}\left[-Y^{*}\right]$. The reverse inequality can be proved similarly.

Example 3 demonstrates that the total profit $Y^{*}$ of a company might belong to $\mathcal{Y}(\rho)$ as a result of natural risk-minimization policy of the company, confirming the practical significance of Problem II. The example can be extended to risk measures $\rho$ of the form

$$
\rho(Y)=\max _{i \in\{1, \ldots, k\}} E\left[Q_{i}(-Y)\right], Q_{1}, \ldots, Q_{k} \in \mathcal{F}_{1}(P) .
$$

Representation of risk measures based on finite number of scenarios (6) is often used for simulation of coherent risk measures in practice, see [25].

The following problem is a version of Problem II for coherent risk measures, continuous from below.

Problem III Let $\rho$ be a coherent risk measure, continuous from below, and let $Y \in \mathcal{L}^{\infty}(\Omega)$. For every $S \in \mathcal{S}(Y)$, the functional $\Lambda_{\rho}(X, Y):=-E[S X]$ is a linear diversifying capital allocation. Which one to choose?

Set $Q$ in (2) can be interpreted as a set of (RadonNikodym derivatives of) possible probabilistic scenarios $Q$, considered by a company. Then $S(Y)$ is a set of Radon-Nikodym derivatives of the "worst-case" scenarios (that is, those ones, for which an equality in (2) holds for a given $Y$ ). Then, capital allocation problem III is interpreted as a problem of choosing the unique probabilistic scenario from a set of worst-case ones, and then assign the capital allocation according to the chosen scenario.

Let $\mathcal{T}$ be the set of all non-empty convex weakly compact subsets of $\mathcal{L}^{1}(\Omega)$. On an abstract level, Problem III is just a problem of choosing the unique element from every $T \in \mathcal{T}$. A map $f: \mathcal{T} \rightarrow \mathcal{L}^{1}(\Omega)$ will be called CA-solution (capital-allocation-solution), if $f(T) \in T, \forall T \in \mathcal{T}$. We will call $f(T)$ the selector from a convex set $T$. Given any CA-solution $f$, the capital allocation can be estimated as

$$
\Lambda_{\rho}(X, Y):=-E[f(\mathcal{S}(Y)) X], \quad \forall X, Y \in \mathcal{L}^{\infty}(\Omega) .
$$




\section{Centroid Capital Allocation}

First, we suggest a CA-solution for the important special case when the probability space $\Omega$ is discrete. Let $\Omega=\left\{\omega_{1}, \ldots, \omega_{n}\right\}$, with $P\left(\omega_{i}\right)=p_{i}>0, i=1, \ldots, n$. Then any r.v. $X$ is just a vector $\left(x_{1}, \ldots, x_{n}\right) \in \mathbb{R}^{n}$ with $x_{i}=X\left(\omega_{i}\right), i=1, \ldots, n$. A discrete probability space typically arise when $x_{n}$ are empirical or Monte Carlo realizations of $X$. In this case, every positively homogeneous and subadditive risk measure $\rho$ (not necessary coherent or continuous from below) can be represented in the form

$$
\rho(Y)=\max _{Q \in Q} E[Q(-Y)], \quad \forall Y \in \mathbb{R}^{n},
$$

for a non-empty convex compact set $Q \subset \mathbb{R}^{n}$. A CAsolution $f$ is a map which assigns a point $f(T) \in T$ to every non-empty convex compact set $T$ in $\mathbb{R}^{n}$.

Example 4 Let $f(T)$ be the center of mass (centroid) of set $T$ given by

$$
f(T)=\frac{\int_{\mathbb{R}^{n}} x I_{T}(x) d x}{\int_{\mathbb{R}^{n}} I_{T}(x) d x},
$$

where $I_{T}(x)$ is the characteristic function of $T$. With $f(T)$ given by (9), formula (7) defines the capital allocation for every positively homogeneous and subadditive risk measure $\rho$. We will call it the centroid capital allocation.

Because $T$ is the set of Radon-Nikodym derivatives of the "worst-case" scenarios, the centroid (9) can be interpreted as the "average", or "expected" element of $T$, assuming that they are "equally probable".

Example 5 For a risk measure $\rho$ given by

$$
\rho(Y)=\max _{i \in\{1, \ldots, k\}} E\left[Q_{i}(-Y)\right], \quad \forall Y \in \mathbb{R}^{n},
$$

where $Q_{1}, \ldots Q_{k}$ are affinely independent vectors in $\mathbb{R}^{n}$, the centroid capital allocation is given by

$\Lambda_{\rho}(X, Y):=E\left[\left(\frac{1}{|I(Y)|} \sum_{i \in I(Y)} Q_{i}\right)(-X)\right], \forall X, Y \in \mathbb{R}^{n}$,

where $I(Y) \subset\{1, \ldots, k\}$ is the set of indexes $i$ such that $E\left[Q_{i}(-Y)\right]=\rho(Y)$.
Detail. In this case, the $\mathcal{S}(Y)$ in (7) is the convex hull of affinely independent vectors $\left\{Q_{i} \mid i \in I(Y)\right\}$, and its centroid is given by $\frac{1}{|I(Y)|} \sum_{i \in I(Y)} Q_{i}$.

Example 6 For a coherent risk measure $\rho$ given by

$$
\rho(Y)=-\inf Y=-\min _{i \in\{1, \ldots, n\}} Y\left(\omega_{i}\right), \quad \forall Y \in \mathbb{R}^{n},
$$

the centroid capital allocation is given by

$$
\Lambda_{\rho}(X, Y):=E[-X \mid Y=\inf Y], \quad \forall X, Y \in \mathbb{R}^{n} .
$$

Detail. In Example 5, let $k=n$, and $Q_{i}\left(\omega_{j}\right)$ is $1 / p_{j}$ for $i=j$ and 0 otherwise, $i=1, \ldots, n ; j=1, \ldots, n$. Then $\rho(Y)$ in (10) takes the form (12), and the centroid capital allocation in (11) takes the form (13).

Next sections discuss what are the desirable properties we want CA-solution to possess, show that the centroid capital allocation satisfies these properties, and also suggest a capital allocation scheme for an arbitrary probability space $\Omega$.

\section{Law-invariant Capital Allocation}

Cherny and Orlov (2011) suggested that the capital allocation should be law-invariant, that is, $\Lambda_{\rho}(X, Y)$ should depend only on the joint law of $(X, Y)$, and have studied such capital allocations for the case when $\rho$ can be represented as

$\rho(Y)=\operatorname{WVaR}_{\mu}(Y)=\int_{0}^{1} \operatorname{CVaR}_{\alpha}(Y) \mu(d \alpha), Y \in \mathcal{L}^{\infty}(\Omega)$,

where $\mu$ is a probability measure on $(0,1]$ and

$$
\mathrm{CVaR}_{\alpha}(Y)=-\frac{1}{\alpha} \int_{0}^{\alpha} q_{Y}(\beta) d \beta, \quad Y \in \mathcal{L}^{\infty}(\Omega) .
$$

Theorem 2.3 in Cherny and Orlov (2011) states that, if the probability space is atomless, and $\rho$ is given by (14), then there exists a unique linear diversifying lawinvariant capital allocation, which is continuous in $X$. In this form, Theorem 2.3 is not applicable for probability spaces with atoms.

Example 7 Let $\Omega=\left\{\omega_{1}, \omega_{2}, \omega_{3}\right\}, \quad p_{i}=P\left(\omega_{i}\right), i=$ $1,2,3, \quad p_{1}<p_{2}<p_{3}, \rho()=.\mathrm{CVaR}_{p_{1}}(),. \quad Y\left(\omega_{1}\right)=$ $Y\left(\omega_{2}\right)<Y\left(\omega_{3}\right)$. Then $\Lambda_{\rho}(X, Y)=a \cdot X\left(\omega_{1}\right)+b \cdot X\left(\omega_{2}\right)$ is a linear diversifying law-invariant and continuous in $X$ capital allocation, for any $a \leq 0, b \leq 0$, such that $a+b=-1$. 
Detail. $\Lambda_{\rho}(X, Y)$ is a valid capital allocation at $Y$ for $\rho($.$) , because \Lambda_{\rho}(Y, Y)=a \cdot Y\left(\omega_{1}\right)+b \cdot Y\left(\omega_{2}\right)=$ $-Y\left(\omega_{1}\right)=\mathrm{CVaR}_{p_{1}}(Y)$. Linearity and continuity is obvious. Also, $\Lambda_{\rho}(X, Y) \leq(a+b) \inf X=-\inf X=$ $\mathrm{CVaR}_{p_{1}}(X)$, thus it is diversifying. Now, at a given probability space, if $\left(X_{1}, Y_{1}\right)$ and $\left(X_{2}, Y_{2}\right)$ have the same joint law, then actually $X_{1}=Y_{1}$ and $X_{2}=Y_{2}$, whence $\Lambda_{\rho}\left(X_{1}, Y_{1}\right)=\Lambda_{\rho}\left(X_{2}, Y_{2}\right)$, and the capital allocation is law-invariant.

However, it follows from the proof of Theorem 2.3 in Cherny and Orlov (2011), that the "law-invariance" condition can be reformulated as follows.

Proposition 2 Let $\rho$ be a coherent risk measure, continuous from below, $Y \in \mathcal{L}^{\infty}(\Omega), S \in \mathcal{S}(Y)$, and $\Lambda_{\rho}(X, Y):=-E[S X]$. The following statements are equivalent.

(i) $\Lambda_{\rho}(X, Y)=\Lambda_{\rho}(E[X \mid Y], Y), \forall X \in \mathcal{L}^{\infty}(\Omega)$

(ii) $S=f(Y)$ a.e. for some measurable $f: \mathbb{R} \rightarrow \mathbb{R}$. In other words, $S$ is $Y$-measurable.

Both these conditions imply that

(iii) $\Lambda_{\rho}\left(X_{1}, Y\right)=\Lambda_{\rho}\left(X_{2}, Y\right)$ whenever pairs $\left(X_{1}, Y\right)$ and $\left(X_{2}, Y\right)$ have the same joint law.

Moreover, if $\Omega$ is atomless, conditions $(i)-(i i i)$ are equivalent.

Proof $(i i) \rightarrow(i): \quad \Lambda_{\rho}(X, Y)=-E[f(Y) X]=$ $-E(E[f(Y) X \mid Y])=-E[f(Y)(E[X \mid Y])]=$ $\Lambda_{\rho}(E[X \mid Y], Y)$.

$(i) \rightarrow(i i): \quad E[S X]=-\Lambda_{\rho}(X, Y)=$ $-\Lambda_{\rho}(E[X \mid Y], Y)=E[S(E[X \mid Y])]=$ $E[(E[S \mid Y]) X], \forall X \in \mathcal{L}^{\infty}(\Omega)$. This implies $S=E[S \mid Y]$, whence $S$ is $Y$-measurable.

$(i) \rightarrow(i i i)$ : Let $X_{1}, X_{2}, Y$ be any r.v.'s such that $\left(X_{1}, Y\right)$ and $\left(X_{2}, Y\right)$ have the same joint law. Then $E\left[X_{1} \mid Y\right]=E\left[X_{2} \mid Y\right]$, hence, $\Lambda_{\rho}\left(X_{1}, Y\right)=$ $\Lambda_{\rho}\left(E\left[X_{1} \mid Y\right], Y\right)=\Lambda_{\rho}\left(E\left[X_{2} \mid Y\right], Y\right)=\Lambda_{\rho}\left(X_{2}, Y\right)$.

The proof of the direction $(i i i) \rightarrow(i i)$ for an atomless probability space can be extracted from the proof of Theorem 2.3 in Cherny and Orlov (2011).

Equivalent conditions (i)-(ii) in Proposition 2 can be interpreted as the "correct" analogue of law-invariance axiom for capital allocations for general probability space $\Omega$. Condition (i) also has the following financial interpretation: the states of natures $\omega_{1}$ and $\omega_{2}$ such that $Y\left(\omega_{1}\right)=Y\left(\omega_{2}\right)$ are indistinguishable from the company point of view, and only average value of $X$ over all such states is taken into account for capital allocation. Theorem 6.3 in Cherny (2006) implies that the corresponding capital allocation exists and unique for the family (14) on general $\Omega$.

Proposition 3 Let $\rho$ be given by (14). Then for every $Y \in \mathcal{L}^{\infty}(\Omega)$, there exists a unique $Y$-measurable element $S^{*} \in \mathcal{S}(Y)$. Moreover, $S^{*}=E[S \mid Y], \forall S \in \mathcal{S}(Y)$.

Proof See Theorem 6.3 in Cherny (2006).

Proposition 3 can be interpreted as a version of Theorem 2.3 in Cherny and Orlov (2011) for general $\Omega$. In particular, in Example 7, there are many law-invariant capital allocations, but only one of them, namely $\Lambda_{\rho}(X, Y)=-\frac{p_{1}}{p_{1}+p_{2}} \cdot X\left(\omega_{1}\right)-\frac{p_{2}}{p_{1}+p_{2}} \cdot X\left(\omega_{2}\right)$, can be represented as $\Lambda_{\rho}(X, Y)=-E[S X]$, where $S \in$ $\mathcal{S}(Y)$ is $Y$-measurable.

Next, we argue that law-invariant capital allocations can be interpreted as ones invariant under automorphisms of the underlying probability space. A bijection $g: \Omega / A_{1} \rightarrow \Omega / A_{2}$ is called mod 0 automorphism of $\Omega$, if (i) $\mathbb{P}\left(A_{1}\right)=\mathbb{P}\left(A_{2}\right)=0$, (ii) $A \in \mathcal{F}$ if and only if $g(A) \in \mathcal{F}$ and (iii) $\mathbb{P}(g(A))=\mathbb{P}(A)$ for all $A \in \mathcal{F}$. Let $\mathcal{A}$ be the set of all mod 0 automorphisms of $\Omega$. Every $g \in \mathcal{A}$ defines a map $B_{g}: \mathcal{L}^{1}(\Omega) \rightarrow \mathcal{L}^{1}(\Omega)$ by $\left(B_{g}(X)\right)(\omega)=X(g(\omega)), \omega \in \Omega / A_{1}$. Obviously, r.v.s $X$ and $B_{g}(X)$ have the same distribution. The converse, in general, does not hold: for $\Omega=(0,1)$ with Lebesgue measure, $X(\omega)=\omega$, and $Y(\omega)=|2 \omega-1|$, $X$ and $Y$ have the same distribution, but $Y \neq B_{g}(X)$ for any $g \in \mathcal{A}$. However, [21, Lemma A.4] implies that a risk measure $\rho: \mathcal{L}^{\infty}(\Omega) \rightarrow \mathbb{R}$, defined on an atomless probability space $\Omega$, and continuous from below, is law-invariant ${ }^{3}$ if and only if $\rho\left(B_{g}(X)\right)=\rho(X), \forall g \in \mathcal{A}$.

This section aims to prove the following result.

Proposition 4 Let $\Omega$ be atomless, and $f: \mathcal{T} \rightarrow \mathcal{L}^{1}(\Omega)$ be a CA-solution such that

(*) $B_{g}(f(T))=f(T)$ whenever $B_{g}(T)=T, g \in \mathcal{A}$.

\section{Then}

\footnotetext{
${ }^{3}$ that is, $\rho(X)=\rho(Y)$ whenever $X$ and $Y$ have the same distribution function
} 
(i) If $\rho$ is a law-invariant coherent risk measure, continuous from below, then $f(\mathcal{S}(Y))$ is $Y$-measurable for every $Y \in \mathcal{L}^{\infty}(\Omega)$, whence $\Lambda_{\rho}(X, Y):=-E[f(S(Y)) X]$ is a law-invariant capital allocation.

(ii) In particular, if $\rho$ is given by (14), $\Lambda_{\rho}(X, Y)$ coincides with the unique linear diversifying lawinvariant continuous in $X$ capital allocation, described in Theorem 2.3 in Cherny and Orlov (2011).

The proof of Proposition 4 requires some preparation. For a fixed $Y \in \mathcal{L}^{1}(\Omega)$, let $\mathcal{A}_{\text {inv }}(Y)=$ $\left\{g \in \mathcal{A} \mid B_{g}(Y)=Y\right\}$.

Proposition 5 Let $\Omega$ be atomless, and $\rho: \mathcal{L}^{\infty}(\Omega) \rightarrow \mathbb{R}$ be a law-invariant coherent risk measure, continuous from below. Then $B_{g}(S(Y))=\mathcal{S}\left(B_{g}(Y)\right), \forall g \in$ $\mathcal{A}$, for every $Y \in \mathcal{L}^{\infty}(\Omega)$. In particular, $B_{g}(S(Y))=$ $\mathcal{S}(Y), \forall g \in \mathcal{A}_{\text {inv }}(Y)$.

Proof Let $g \in \mathcal{A}$ and $S \in \mathcal{S}(Y)$. Because $S$ and $B_{g}(S)$ have the same distribution, $B_{g}(S) \in \mathcal{S}$ by [10, corollary 4.3]. Because

$$
\begin{aligned}
& \rho\left(B_{g}(Y)\right)=\rho(Y)=-E[S Y]=-\int_{\Omega} S(\omega) Y(\omega) d \mathbb{P}(\omega) \\
& =-\int_{\Omega} S(g(\omega)) Y(g(\omega)) d \mathbb{P}(\omega)=-E\left[B_{g}(S) B_{g}(Y)\right],
\end{aligned}
$$

this implies $B_{g}(S) \in \mathcal{S}\left(B_{g}(Y)\right)$, or $B_{g}(S(Y)) \subseteq$ $\mathcal{S}\left(B_{g}(Y)\right)$. Thus,

$$
\begin{aligned}
\mathcal{S}(Y) & =B_{g^{-1}}\left(B_{g}(S(Y))\right) \subseteq B_{g^{-1}}\left(\mathcal{S}\left(B_{g}(Y)\right)\right) \\
& \subseteq \mathcal{S}\left(B_{g^{-1}}\left(B_{g}(Y)\right)\right)=\mathcal{S}(Y)
\end{aligned}
$$

where the first and last equalities follows from the fact that $g$ is a bijection. This shows that actually $B_{g}(\mathcal{S}(Y))=\mathcal{S}\left(B_{g}(Y)\right)$.

Proposition 6 Let $\Omega$ be atomless, and $\rho: \mathcal{L}^{\infty}(\Omega) \rightarrow \mathbb{R}$ be a law-invariant coherent risk measure, continuous from below, $Y \in \mathcal{L}^{\infty}(\Omega)$, and $X \in \mathcal{S}(Y)$. Then $X$ and $Y$ are comonotone, that is, there exists a set $E \subseteq \Omega$ such that $\mathbb{P}[E]=1$ and $\left(X\left(\omega_{1}\right)-X\left(\omega_{2}\right)\right)\left(Y\left(\omega_{1}\right)-\right.$ $\left.Y\left(\omega_{2}\right)\right) \geq 0$ for all $\omega_{1}, \omega_{2} \in E$.

Proof [10, Lemma 4.3] implies that there exists an r.v. $Z$ with the same distribution as $X$ which is comonotone with $Y$. Then $Z \in S$, where $S$ defined in (3), by [10, corollary 4.3]. Thus $E[Z Y] \leq \rho(Y)=E[X Y]$, whence $X$ is comonotone with $Y$ by [15, Theorem A.24].

Proposition 7 Let $\Omega$ be atomless, and $X, Y \in \mathcal{L}^{1}(\Omega)$ be comonotone r.v.s. Then $X$ is $Y$-measurable if and only if $\mathcal{A}_{\text {inv }}(Y) \subseteq \mathcal{A}_{\text {inv }}(X)$

Proof If $X$ is $Y$-measurable, $X=f(Y)$ for some $f$ : $\mathbb{R} \rightarrow \mathbb{R}$, and $\left(B_{g}(X)\right)(\omega)=X(g(\omega))=f(Y(g(\omega)))=$ $f\left(\left(B_{g}(Y)\right)(\omega)\right)=f(Y(\omega))=X(\omega), \forall g \in \mathcal{A}_{\text {inv }}(Y)$ for almost all $\omega \in \Omega$, and "only if" follows. Let us prove the "if" direction. Let $E=\{\omega \in \Omega \mid \mathbb{P}[Y=Y(\omega)]>0\}$. Because $X$ and $Y$ are comonotone, it is sufficient to show that $X$ is $Y$-measurable on $E$. If not, there exists constants $x, y \in \mathbb{R}$ such that $\mathbb{P}\left[I_{<}\right]>0$ and $\mathbb{P}\left[I_{>}\right]>0$, where $I_{<}=\{\omega \in \Omega \mid X(\omega)<x, Y(\omega)=y\}$ and $I_{>}=$ $\{\omega \in \Omega \mid X(\omega)>x, Y(\omega)=y\}$. Because the probability space is atomless, we can find two subsets $I_{1} \in I_{<}$ and $I_{2} \in I_{>}$such that $\mathbb{P}\left[I_{1}\right]=\mathbb{P}\left[I_{2}\right]>0$. Let us show that there exists an $g \in \mathcal{A}$ such that $g\left(I_{1}\right)=I_{2}$ a.s. and $g(\omega)=\omega \forall \omega \notin I_{1} \cup I_{2}$, which would imply $g \in \mathcal{A}_{\text {inv }}(Y)$ but $g \notin \mathcal{A}_{\text {inv }}(X)$, a contradiction. Because $\Omega$ is atomless (and standard), we can assume that $\Omega=(0,1)$ with Lebesgue measure. Then $I_{1}, I_{2}$ are Lebesgue measurable, and, ignoring the sets of measure 0, we may assume that they are Borel sets. Then for almost every $\omega \in I_{1}$ we can find $g(\omega) \in I_{2}$ defined by relation $\mathbb{P}\left((0, \omega) \cap I_{1}\right)=\mathbb{P}\left((0, g(\omega)) \cap I_{2}\right)$.

Proof of Proposition 4 If $\rho$ is a law-invariant coherent risk measure, continuous from below, then $B_{g}(\mathcal{S}(Y))=\mathcal{S}(Y), \forall g \in \mathcal{A}_{\text {inv }}(Y)$ by Proposition 5. This implies that $B_{g}(f(\mathcal{S}(Y)))=f(\mathcal{S}(Y)), \forall g \in \mathcal{A}_{\text {inv }}(Y)$, which is equivalent to saying that $f(S(Y))$ is $Y$ measurable by Propositions 6 and 7, and (i) follows. Now (ii) follows from Theorem 2.3 in Cherny and Orlov (2011).

Condition (*) in Proposition 4 reformulates the "law-invariance" axiom of Cherny and Orlov (2011) as follows: the selector $f(T)$ from any convex set $T$ should be a fixed point of any map from the family $B_{g}, g \in \mathcal{A}$, mapping $T$ into $T$. The interpretation of this condition is straightforward: if the risk measure $\rho$ is law-invariant, then the corresponding set $\mathcal{S}(Y)$ is invariant under any automorphism of an underlying probability space, and we require that the resulting capital allocation should also be invariant under such 
automorphisms. However, some popular law-invariant coherent risk measures, including $\rho_{M A D}$ from Example 1 , can not be represented in the form (14), and a lawinvariant capital allocation may still be non-unique, see Cherny and Orlov (2011) for more examples. Also, a law-invariance axiom for capital allocation does not make sense if $\rho$ is not law-invariant (which is typically the case in Example 2). In the next sections, we develop an approach to capital allocation, valid for all coherent risk measures, by extending the condition $(*)$ to a larger family of maps, called affine isometries.

\section{Capital Allocation and Center of a Convex Set}

Let $X$ be a Banach space with norm $\|$.$\| . A map B$ : $X \rightarrow X$ is called an isometry if $\|B(x)-B(y)\|=\| x-$ $y \|, \forall x, y \in X$. A map $B$ is called linear, if $B(x+y)=$ $B(x)+B(y), \forall x, y \in X$, and $B(\alpha \cdot x)=\alpha \cdot B(x), \forall x \in$ $X, \alpha \in \mathbb{R}$. A map $B$ is affine if $B(x)-B(y)=A(x-$ $y), \forall x, y \in X$ for some linear map $A$. Equivalently, a map $B$ is affine if and only if it preserves the centers of mass:

$$
\begin{aligned}
& B\left(\sum_{i=1}^{n} \alpha_{i} x_{i}\right)=\sum_{i=1}^{n} \alpha_{i} B\left(x_{i}\right), \\
& \forall x_{1}, \ldots, x_{n} \in X, \alpha_{1}, \ldots, \alpha_{n} \in \mathbb{R}, \sum_{i=1}^{n} \alpha_{i}=1 .
\end{aligned}
$$

It is straightforward to verify, that for every $A \in \mathcal{A}$, the map $B_{A}$ defined as $\left(B_{A}(X)\right)(\omega)=X(A(\omega)), \omega \in \Omega$ is a linear (and hence affine) isometry over Banach space $\mathcal{L}^{1}(\Omega)$. Thus, condition (*) in Proposition 4 is just a special case of the following one.

Definition 2 (AI-preserving CA-solution). An CAsolution $f: \mathcal{T} \rightarrow \mathcal{L}^{1}(\Omega)$ will be called AI-preserving (affine-isomerty-preserving), if $B(f(T))=f(T)$ for every affine isometry $B$ such that $B(T)=T$. In other words, $f$ is an AI-preserving CA-solution, if the selector $f(T)$ from any convex set $T$ is a fixed point of any affine isometry, mapping $T$ into $T$. In this case, we will call $f(T)$ the center of convex set $T$.

For a finite probability space, AI-preserving CAsolution is presented in Example 4.
Proposition 8 If probability space $\Omega$ is a finite set, then the centroid capital allocation, defined in Example 4, is an AI-preserving CA-solution.

Proof It follows from (16) that the centroid (9) is a fixed point of any affine isometry, mapping $T$ into $T$.

From now, we assume that $\Omega$ is an infinite set. In this case, the notion of centroid for subsets of $\mathcal{L}^{1}(\Omega)$ does not exist in the form (9), because there is no analogue of Lebesgue measure on an infinitedimensional separable Banach space. More precisely, every translation-invariant measure on such space that is not identically zero assigns infinite measure to all open subsets, see Hunt et al. (1992). For this reason, the construction of the "natural" center for every convex weakly compact subset $T$ of a Banach space is a non-trivial and well-known problem. Brodskii and Milman (1948) developed a method for assigning center to every set $T$ with "normal structure", see their paper for definition. The construction which works in general case and returns a unique center was suggested 33 years later in Lim (1981).

To be self-contained, we repeat the construction here. It defines $C_{\alpha}$ for all ordinals $\alpha$ by transfinite induction. Let $C_{0}=T$. Let $\beta$ be an ordinal and suppose that $C_{\alpha}$ has been defined for $\alpha<\beta$. If $\beta$ is a limit ordinal, let $C_{\beta}=\bigcap_{\alpha<\beta} C_{\alpha}$. Otherwise, let $C_{\beta}$ be a closed convex hull of the set

$$
\begin{aligned}
& \left\{z \in C_{\gamma}: z=\frac{1}{2}(x+y),\right. \\
& \text { for some } \left.x, y \in C_{\gamma} \text { with }\|x-y\|=\frac{1}{2} \operatorname{diam} C_{\gamma}\right\} \text {, }
\end{aligned}
$$

where $\gamma$ is the predecessor of $\beta$, and $\operatorname{diam} C_{\gamma}=$ $\sup _{x, y \in C_{\gamma}}|| x-y \|$. Lim proved that, for sufficiently large ordinals $\beta, C_{\beta}$ are identical, and consist of exactly one point, which he called the center of $T$. This construction defines the CA-solution, which we denote $f^{*}$, and the corresponding capital allocation in (7) will be denoted $\Lambda_{\rho}^{*}(X, Y)$.

Proposition 9 [Theorem 1 in Lim (1981)] Let $T$ be a nonempty weakly compact convex subset of a Banach space. The center $f^{*}(T)$ is a fixed point of every affine isometry mapping $T$ into $T$.

In other words, Proposition 9 states that $f^{*}$ is an AIpreserving CA-solution.

We summarize the discussion above as follows. 
- $\Lambda_{\rho}^{*}(X, Y)$ is linear and diversifying.

- $\Lambda_{\rho}^{*}(X, Y)$ coincides with the directional derivative (1), provided that it exists.

- If $\rho$ is law-invariant, $\Lambda_{\rho}^{*}(X, Y)$ is law-invariant. In particular, it coincides with the capital allocation described in Theorem 2.3 of Cherny and Orlov (2011), if $\Omega$ is atomless, and $\rho$ belongs to the family (14).

- However, $\Lambda_{\rho}^{*}(X, Y)$ is defined for every coherent risk measure $\rho$, continuous from below (and for all $X, Y \in \mathcal{L}^{\infty}(\Omega)$ ).

In particular, we are now able to solve the capital allocation problem for the risk measures in Examples 1 and 2 in the "difficult" case $Y \in \mathcal{Y}(\rho)$. We remark that, although the original construction of the center uses ordinals and is not explicit, Proposition 9 can be used to derive the formulas for $\Lambda_{\rho}^{*}(X, Y)$ easily, at least in the examples below.

Example 8 Let $\rho$ be a coherent risk measure given by (4), and $Y$ be such that $E_{Q_{1}}[-Y]=E_{Q_{2}}[-Y]$. Then

$$
\Lambda_{\rho}^{*}(X, Y)=E_{\left(Q_{1}+Q_{2}\right) / 2}[-X], \quad \forall X \in \mathcal{L}^{\infty}(\Omega) .
$$

Detail. In this case, $S(Y)=\left\{S_{\alpha} \mid \alpha \in[0,1]\right\}$, where $S_{\alpha}=\alpha \frac{d Q_{1}}{d P}+(1-\alpha) \frac{d Q_{2}}{d P}$. The map $S_{\alpha} \rightarrow S_{1-\alpha}$ is an affine isometry, mapping $\mathcal{S}(Y)$ to $\mathcal{S}(Y)$, thus the center is the fixed point of this isometry, $S_{1 / 2}$.

Example 9 Let $\rho(Y)=c\|Y-E Y\|_{1}-E Y, c \in\left[0, \frac{1}{2}\right]$, see Example 1. For any nonconstant $Y \in \mathcal{L}^{\infty}(\Omega)$,

$$
\begin{array}{r}
\Lambda_{\rho}^{*}(X, Y)=E[-X]+c E[X-E X \mid Y>E Y] \mathbb{P}[Y>E Y] \\
\quad-c E[X-E X \mid Y<E Y] \mathbb{P}[Y<E Y], \quad \forall X \in \mathcal{L}^{\infty}(\Omega) .
\end{array}
$$

Detail. For $c \in\left(0, \frac{1}{2}\right]$ and nonconstant $Y, S(Y)=$ $1+c(E Z-Z), Z \in \operatorname{sign}[Y-E Y]$, see Example 1. Let $\mathcal{B}_{Y}$ be a set of maps $B: \mathcal{L}^{\infty}(\Omega) \rightarrow \mathcal{L}^{\infty}(\Omega)$, such that $B(X)=1+A(X-1)$, where $(A(X))(\omega)=-X(\omega)$ if $Y(\omega)=E Y$, and $(A(X))(\omega)=X(\omega)$ otherwise. Then $B \in \mathcal{B}_{Y}$ are affine isometries, which maps $\mathcal{S}(Y)$ into itself. Indeed, $A(Z) \in \operatorname{sign}[Y-E Y]$ if and only if $Z \in \operatorname{sign}[Y-E Y]$, whence $B(S)=1+c(E Z-Z)=1+$ $A(c(E Z-Z))=1+c(E A(Z)-A(Z)) \in \mathcal{S}(Y)$ for all $S \in \mathcal{S}(Y)$. Thus, the center $S^{*} \in \mathcal{S}(Y)$ is a fixed point of every $B \in \mathcal{B}_{Y}$, which implies $S^{*}=1+c(E Z-Z)$ for the unique $Z \in \operatorname{sign}[Y-E Y]$ such that $Z(\omega)=0$ if $Y(\omega)=E Y$, and (18) follows.

If $Y=C$ is a constant, the capital allocation in Example 9 is given by

$$
\Lambda_{\rho}^{*}(X, C)=E[-X] .
$$

Relation (19) is a corollary from Proposition 4 (i), and remains valid for every law-invariant coherent risk measure $\rho$, which is continuous from below and can be defined on an atomless probability space.

Next, we remark that our methodology can be easily extended to wider families of risk measures. Corollary 5.1. in Kalkbrener (2005) solves the capital allocation problem for nonconstant $Y$ for risk measure $\rho_{S T D}(X)=c \cdot \sigma(X)-E X$, which is not coherent (R1 fails). Similarly, $\mathrm{R} 1$ fails for $\rho(Y)=c\|Y-E Y\|_{1}-E Y$ in Example 9 if $c>\frac{1}{2}$. Rockafellar et al. (2006a) showed that every continuous risk measure satisfying $\mathrm{R} 2-\mathrm{R} 4$ can still be represented in the form

$$
\rho(Y)=\max _{S \in \mathcal{S}} E[S(-Y)], \text { for all } Y \in \mathcal{L}^{\infty}(\Omega),
$$

where $S$ is nonempty, closed, convex set such that $E S=1, \forall S \in \mathcal{S}$ ( $\rho$ is coherent if and only if $S \geq 0, \forall S \in$ $S$ ), whence the capital allocation problem reduces to the choice of the center in a set $\mathcal{S}(Y)$ of maximizers in (20). In case $\rho=\rho_{S T D}, \mathcal{S}(Y)$ is a singleton for nonconstant $Y$, and $\mathcal{S}(Y)=\mathcal{S}$ for constants. In the latter case, allocation formula (19) remains valid, complementing Corollary 5.1. in Kalkbrener (2005). Similarly, capital allocation (18) in Example 9 remains valid for $c>\frac{1}{2}$.

A coherent risk measure $\rho(Y)=-\inf Y$, which, in general, is not continuous from below, can be represented in the form (2), but with sup instead of max, and the supremum is not attained. However, Proposition 4.14 in Föllmer and Shield (2004) states that every coherent risk measure $\rho$ can be represented in the form

$$
\rho(Y)=\sup _{Q \in Q} E_{Q}[-Y],
$$

for some subset $Q$ of the Banach space $b a(\Omega)=$ $b a(\Omega, \mathcal{F}, \mathbb{P})$ of finitely additive set functions on $(\mathcal{F}, \mathbb{P})$ with bounded variation, absolutely continuous with respect to $P$, see eg. appendix A.6 in Föllmer and Shield (2004) for definitions and the meaning of $E_{Q}[-X]$. Actually, for coherent $\rho, Q \subset b a^{1}(\Omega)$, where $b a^{1}(\Omega) \subset$ 
$b a(\Omega)$ is a set of nonnegative set functions $Q$ normalized as $Q(\Omega)=1$. Moreover, $Q$ can be chosen as a convex set for which the supremum in (21) is attained. Thus, one can find the capital allocation as

$$
\Lambda_{\rho}^{*}(X, Y)=E_{Q^{*}}[-X], \text { for all } X \in \mathcal{L}^{\infty}(\Omega),
$$

where $Q^{*}$ is the center of the set $Q(Y)$ of maximizers in (21).

Actually, representation (21) remains valid for every positively homogeneous and subadditive $\rho$, which is lower-semicontinuous, that is, set $\left\{Y \in \mathcal{L}^{\infty}(\Omega) \mid \rho(Y) \leq\right.$ $C\}$ is closed for all $C \in \mathbb{R}$, see eg. Rudloff (2006). In addition to coherent risk measures, there are other interesting families of functionals with these properties (see Grechuk and Zabarankin (2012b)).

Definition 3 (deviation measures and error measures). A positively homogeneous, subadditive, and lower-semicontinuous functional $\rho: \mathcal{L}^{\infty}(\Omega) \rightarrow \mathbb{R}$ is called

- $a$ deviation measure, if $\rho(Y)=0$ for constant $Y$, but $\rho(Y)>0$ otherwise;

- an error measure, if $\rho(0)=0$, but $\rho(Y)>0$ for $Y \neq 0$.

Deviation measures introduced in Rockafellar et al. (2006a) to measure the non-constancy, inherent in an r.v. $Y$. If $\rho$ is a deviation measure, $\Lambda_{\rho}^{*}(X, Y)$ should be interpreted as deviation contribution (rather than risk contribution), that is, it measures the contribution of nonconstancy of $X$ to the nonconstancy of $Y$. The capital allocation problem with deviation measures naturally arises in the context of cooperative portfolio investment, see Grechuk et al. (2013). The solution of the form $\Lambda_{\rho}(X, Y)=E_{Q}[-X], Q \in Q(Y)$ was proposed in Grechuk and Zabarankin (2012a), but the issue that $Q(Y)$ might not be a singleton was ignored.

Error measures are designed to measure nonzeroness of an r.v., with the prominent example $\rho(X)=$ $\|X\|_{2}$, see Rockafellar et al. (2008). If $\rho$ is an error measure, a natural interpretation for $\Lambda_{\rho}^{*}(X, Y)$ is error contribution. For example, assume that there are $n$ sources of random noise $X_{1}, X_{2}, \ldots, X_{n}$, with total noise $Y=\sum_{i=1}^{n} X_{i}$, and we need to estimate the contribution of each source to the total noise in the system. This may be useful e.g. for deciding which source of noise should be removed/reduced first.

\section{Discussion}

We argue that the problem of selection the unique linear diversifying capital allocation (Problem II) is essentially a problem of selection the unique probabilistic scenario from a set of worst-case ones (Problem III), and then the capital should be allocated according to the selected scenario via (7). The most straightforward approach would be assume that all these scenarios are "equally probable", and then calculate the "average" one. If $\Omega$ is finite, this leads to the centroid capital allocation (9).

The assumption that $\Omega$ is finite is not as unrealistic as it seems, because discrete distributions naturally arise when historical data is used to estimate the future outcomes. For example, assume that the company makes an investment to the stock market, and solves a (one period) portfolio optimization problem in the form

$$
\min _{X \in \mathcal{V}} \rho(X) \quad \text { subject to } \quad E X \geq \pi,
$$

where $\pi$ is a desired expected return, and $\mathcal{V}$ is a feasible set of portfolio returns $X$, given by

$\mathcal{V}=\left\{X \mid X=\sum_{j=0}^{m} r_{j} x_{j}, \sum_{j=0}^{m} x_{j}=1,\left(x_{0}, \ldots, x_{m}\right) \in \mathbb{R}^{m+1}\right\}$,

where $r_{0}, r_{1}, \ldots, r_{m}$ are the rates of return of $m+1$ assets, of which asset 0 is risk-free, $x_{j}$ is the fraction of capital invested into asset $j$ (with $x_{j}<0$ corresponding to short selling), and $\sum_{j=0}^{m} x_{j}=1$ is the budget constraint. Obviously, future rates of return are unknown, and can be estimated using Monte-Carlo simulation or historical data. As a result, one get $T \geq 2$ (usually equiprobable) scenarios with the return $r_{j i}$ for $r_{j}$ under scenario $i$. In this model, each $Y \in \mathcal{V}$ is a discrete r.v. assuming values $y_{1}, \ldots, y_{T}$ with equal probabilities, and the probability space can be assumed to have $T$ elements.

Now, if a company, consisting of $n$ subsidiaries, invests in a portfolio $Y^{*} \in \mathcal{V}$, each subsidiary investing to portfolio $X_{i}$, with $Y^{*}=\sum_{i=1}^{n} X_{i}$, the capital allocation problem arises, and the centroid capital allocation (Example 4) presents a solution: risk contribution of subsidiary $i$ is given by $k_{i}=\Lambda_{\rho}\left(X_{i}, Y\right)$, where $\Lambda_{\rho}$ is given by (7) with $f$ defined in (9).

If the underlying distributions are continuous, and $\Omega$ cannot be modelled as finite probability space, the 
set of (the Radon-Nikodym derivatives of) worst-case probabilistic scenarios $\mathcal{S}(Y)$ is infinite-dimensional, and we cannot say that these scenarios are "equally probable", because there is no reasonable way to introduce a probability measure on $\mathcal{S}(Y)$.

If the underlying risk measure (and whence the set $\mathcal{S}(Y)$ ) has any kind of "symmetry" (which we mathematically formalised as invariance under affine linear transformations), we suggest that the capital allocation scheme $\Lambda$ should possess the same kind of symmetry. For example, if $\rho$ is law-invariant, $\Lambda$ should be lawinvariant, consistently with Cherny and Orlov (2011). As another example, risk measure $\rho_{M A D}$ (see Example 9) equally penalises ups and downs comparing to mean, in sense that $\rho_{M A D}(X)=\rho_{M A D}(Y)$ if $E X=E Y$ and $X-E X=E Y-Y$; in particular heavy down tails in penalized to the same extend as heavy up tails. We require that the same property should be reflected in the capital allocation scheme, that is, heavy down tail should not be a reason for extra capital allocation, comparing to heavy up tail. All this kind of informal reasoning is summarised, formalised, and generalised in Definition 2: if an affine isometry does not change the risk measure, it also should not change the capital allocation. A construction of Lim (1981) returns the capital allocation with this property.

This construction, however, is not explicit, and cannot be used directly in practical applications. Example 9 shows how the use of a particular affine isometries may lead to a quick solution. In case when such isometries are not available, one may approximate the underlying distributions with the discrete ones, and use the centroid capital allocation (9), which can be efficiently computed.

Example 10 (numerical illustration) A company invests its capital in a portfolio with $m=4$ risky assets: Lloyds Banking Group PLC, BT Group PLC, COCACOLA HBC N, and Imperial Tobacco Group PLC. We identify $T=52$ scenarios with assets' weekly rates of returns from 9-September-2013 to 8-September-2014, that is, $r_{j i}, j=1,2,3,4, i=1, \ldots, 52$, is the historical rate of return of asset $j$ during week $i$. With risk measure $\rho(X)=\frac{1}{2}\|X-E X\|_{1}-E X$, see Example 9, and $\pi=0.003$, the optimal portfolio in (23) is $Y^{*}=$ $\sum_{j=1}^{m} x_{j}^{*} r_{j} \approx 0.166 r_{1}+0.351 r_{2}+0.037 r_{3}+0.446 r_{4}$. In this case, $\mathbb{P}\left[Y^{*}=E Y^{*}\right]=\frac{2}{52}>0$, hence $Y^{*} \in \mathcal{Y}(\rho)$, see Example 1, and linear diversifying capital allo- cation is not unique. In contrast, the capital allocation defined in Section 5 is unique and is given by (18). In particular, with $n=4$, and $X_{j}=x_{j}^{*} r_{j}$, $j=1, \ldots, 4,(18)$ implies that relative risk contributions $\Lambda_{\rho}^{*}\left(X_{j}, Y^{*}\right) / \rho\left(Y^{*}\right)$ of assets $j=1,2,3,4$ into the portfolio are about $23 \%, 33 \%, 8 \%, 36 \%$, correspondingly.

\section{An Application to Optimal Risk Sharing}

This section applies the developed capital allocation technique to a related problem of optimal risk sharing, an old and classical problem originated by Borch (1962), Arrow (1963), and others. There are $n$ agents, indexed by $I=\{1,2, \ldots, n\}$. Agent $i \in I$ has an initial endowment $Y_{i} \in \mathcal{L}^{\infty}(\Omega)$ and associated risk measure $\rho_{i}: \mathcal{L}^{\infty}(\Omega) \rightarrow \mathbb{R}$. The total endowment $Y=\sum_{i=1}^{m} Y_{i}$ is then redistributed among agents in attempt to reduce their risk. An allocation $\vec{X}=\left(X_{1}, X_{2}, \ldots, X_{n}\right)$, where $X_{i}$ is the share of agent $i \in I$, is called attainable if $\sum_{i=1}^{m} X_{i}=Y$. An attainable allocation is called Pareto optimal if there are no attainable allocation $\vec{Z}=\left(Z_{1}, Z_{2}, \ldots, Z_{n}\right)$ with $\rho_{i}\left(Z_{i}\right) \leq \rho_{i}\left(X_{i}\right), i \in I$, with at least one inequality being strict. The optimal risk sharing problem can be divided into two steps.

\section{S1. Find all Pareto optimal allocations;}

S2. If there are many, choose a "fair" one.

We will restrict our attention to S2. A classical approach (see eg. Aase (2002) for a survey) is to allow agents to trade and reach the equilibrium. A price is a linear continuous functional $P: \mathcal{L}^{\infty}(\Omega) \rightarrow \mathbb{R}$, such that $P(1)=1$ and $P(X) \geq 0$ whenever $X \geq 0$. Any price can be represented in the form $P(X)=E_{Q}[X], \forall X \in$ $\mathcal{L}^{\infty}(\Omega)$, for some $Q \in b a^{1}(\Omega)$, which is called pricing rule. For agent $i \in I$, let $X_{i}(Q)$ be an optimal endowment of agent $i$ under $Q$, that is, a minimizer in the problem

$$
\min _{X_{i} \in \mathcal{L}^{\infty}(\Omega)} \rho_{i}\left(X_{i}\right) \quad \text { s.t. } \quad E_{Q}\left[X_{i}\right] \leq E_{Q}\left[Y_{i}\right], \quad i \in I .
$$

If $\sum_{i=1}^{m} X_{i}(Q)=Y, Q$ is called equilibrium pricing rule (EPR), and $\vec{X}(Q)=\left(X_{1}(Q), X_{2}(Q), \ldots, X_{n}(Q)\right)$ is equilibrium allocation. The classical First Welfare Theorem, see eg. Becker (1991), states that, under very 
general conditions on risk measures, every equilibrium allocation $\vec{X}(Q)$ is Pareto optimal. Thus, if EPR $Q^{*}$ exists and unique, $\vec{X}\left(Q^{*}\right)$ is a natural solution of $\mathrm{S} 2^{4}$. If, however, EPR is not unique, $\mathrm{S} 2$ reduces to the following problem.

Problem IV Let $\mathcal{P}=\left\{\rho_{1}, \rho_{2}, \ldots, \rho_{n}, Y_{1}, Y_{2}, \ldots, Y_{n}\right\}$ be a risk sharing problem, and $\mathcal{E}_{P} \subset b a^{1}(\Omega)$ be the set of all EPRs. Assume that $\mathcal{E}_{\mathcal{P}}$ is non-empty, but not a singleton. Which $Q \in \mathcal{E}_{\mathcal{P}}$ to choose?

Recently, optimal risk sharing problem has been intensively studied in the case when $\rho_{i}$ are coherent (see Burgert and Ruschendorf (2008)), or, more generally, convex ${ }^{5}$ risk measures, see Heath and $\mathrm{Ku}$ (2004), Jouini et al. (2008), Filipovic and Kupper (2008), Dana and Le Van (2010). In these papers, the existence of Pareto optimal and equilibrium allocation has been investigated, but the Problem IV has not been addressed.

We argue that there is a close connection between Problem IV and capital allocation problem, formulated in the introduction, at least in coherent case. First, if $\rho_{i}$ satisfy R4, and $\vec{X}$ is Pareto optimal, then, for any constants $C_{i} \in \mathbb{R}$, with $\sum_{i=1}^{m} C_{i}=0$, the allocation

$$
\left(X_{1}+C_{1}, X_{2}+C_{2}, \ldots, X_{n}+C_{n}\right)
$$

is also Pareto optimal (see eg. Jouini et al. (2008)), and S2 reduces to choosing a $C=\left(C_{1}, C_{2}, \ldots, C_{n}\right) \in \mathbb{R}^{n}$, which can be called capital reallocation. $C_{i}$ can be considered as fair premium for investor $i$ for taking share $X_{i}$ of a total risk $X$, which is, intuitively, very close to risk contribution of $X_{i}$ to $X$.

This intuition can be made precise if $\rho_{i}$ are coherent risk measures, given in the form (21) for $Q^{i} \subset b a^{1}(\Omega)$, $i \in I$. In this case, the risk sharing problem $\mathcal{P}$ will be called coherent. Theorem 2.8 in Burgert and Ruschendorf (2008) states, that, for coherent $\mathcal{P}$, an equilibrium exists if and only if $Q^{*}=\bigcap_{i=1}^{n} Q^{i}$ is non-empty. The coherent risk measure $\rho^{*}$, corresponding to $Q^{*}$ via (21), can be written as infimal convolution

$$
\rho^{*}(Y)=\min _{\vec{X}: \Sigma X_{i}=Y}\left(\rho_{1}\left(X_{1}\right)+\ldots+\rho_{n}\left(X_{n}\right)\right),
$$

\footnotetext{
${ }^{4}$ Formally, different equilibrium allocations $\vec{X}\left(Q^{*}\right)$ may correspond to the same EPR $Q^{*}$. We will call allocations $\vec{X}^{1}=\left(X_{1}^{1}, X_{2}^{1}, \ldots, X_{n}^{1}\right)$ and $\vec{X}^{2}=\left(X_{1}^{2}, X_{2}^{2}, \ldots, X_{n}^{2}\right)$ equivalent, if $\rho_{i}\left(X_{i}^{1}\right)=\rho_{i}\left(X_{i}^{2}\right), i \in I$; the "unique" solution to $\mathrm{S} 2$ should be understood as "unique up to equivalence".

${ }^{5} \mathrm{~A} \rho: \mathcal{L}^{\infty}(\Omega) \rightarrow \mathbb{R}$ is a convex risk measure if it is convex and satisfies R1 and R4 in Definition 1
}

and describes the optimal total risk with respect to all attainable allocations. Theorem 3.13 in Filipovic and Kupper (2008) states that, for every Pareto optimal $\vec{X}$, allocation (26) is an equilibrium for $C_{i}=$ $E_{Q}\left[Y_{i}-X_{i}\right], i \in I$, for every $Q \in Q(Y)$, where $Q(Y)$ is the set of maximizers in (21) for $\rho^{*}$. Thus, in this case, $\mathcal{E}_{\mathcal{P}}=Q(Y)$, and the Problem IV is exactly equivalent to choosing an element $Q \in Q(Y)$, that is, to capital allocation problem with risk measure $\rho^{*}$. In particular, the equilibrium is unique if and only if the directional derivative (1) exists for $\rho=\rho^{*}$.

Thus, for every coherent risk sharing problem $\mathcal{P}$, such that $Q^{*}$ is non-empty, our capital allocation scheme returns the unique equilibrium given by (26) with

$$
C_{i}=E_{Q^{*}}\left[Y_{i}-X_{i}\right]=\Lambda_{\rho^{*}}^{*}\left(X_{i}-Y_{i}, Y\right), \quad Q^{*}=f^{*}\left(\mathcal{E}_{\mathcal{P}}\right),
$$

where $f^{*}$ and the corresponding $\Lambda^{*}$ are defined in Section 5 .

In general, by solution of Problem IV we mean the scheme returning the unique EPR $Q(P)$ for every risk sharing problem $\mathcal{P}$ such that $\mathcal{E}_{\mathcal{P}} \neq \emptyset$. We call the solution consistent, if $Q\left(\mathcal{P}_{1}\right)=Q\left(\mathcal{P}_{2}\right)$ whenever $\mathcal{E}_{\mathcal{P}_{1}}=\mathcal{E}_{\mathcal{P}_{2}}$. A natural extension of (28) to general case is the solution given by

$$
Q(\mathcal{P})=f^{*}\left(\mathcal{E}_{\mathcal{P}}\right), \quad \forall \mathcal{P},
$$

where $f^{*}$ is defined in Section 5.

Proposition 10 (29) is a consistent solution of Problem IV, coinciding with (28) for coherent $\mathcal{P}$. It returns the unique equilibrium (and whence the unique Pareto optimal allocation) for any (not necessary coherent) risk sharing problem $\mathcal{P}$ with non-empty and weakly compact $\mathcal{E}_{P \text {. }}$.

Proof An r.v. $\lambda X_{i}(Q)+(1-\lambda) X_{i}\left(Q_{2}\right)$ is a minimizer in (25) for $Q_{\lambda}=\lambda Q_{1}+(1-\lambda) Q_{2}$, whence $Q_{\lambda} \in \mathcal{E}_{P}$ whenever $Q_{1}, Q_{2} \in \mathcal{E}_{\mathcal{P}}$, thus $\mathcal{E}_{P}$ is convex, and $f^{*}\left(\mathcal{E}_{\mathcal{P}}\right)$ is well-defined.

The set $\mathcal{E}_{\mathcal{P}}$ is non-empty, if, for example, $\Omega$ is atomless and all $\rho_{i}$ are law-invariant convex risk measures, see Corollary 3.8 in Filipovic and Kupper (2008).

Example 11 Let $n=2, \mathcal{P}=\left\{\rho_{1}, \rho_{2}, Y_{1}, Y_{2}\right\}$, where $\rho_{1}$ is given by (15) for some $\alpha \in(0,1), \rho_{2}(X):=$ $-E X+\lambda E\left[[X-E X]_{-}^{p}\right]^{1 / p}, \lambda \in[0,1], p \in[1, \infty]$, and 
$Y=Y_{1}+Y_{2}$ is continuous (here $[X]_{-}=\max \{0,-X\}$ ). Proposition 3.2 in Jouini et al. (2008) states that all Pareto optimal allocations in $\mathcal{P}$ are given by $\left(X_{1}+C, X_{2}-C\right), C \in \mathbb{R}$, where $X_{1}=-[Y-K]_{-}, X_{2}=$ $\max \{Y, K\}$, for some constant $K=K(\alpha, \lambda, p)$. According to (28), the constant $C$ should be chosen as

$C=\Lambda_{\rho^{*}}^{*}\left(X_{1}-Y_{1}, Y\right)=\lim _{\varepsilon \rightarrow 0} \frac{\rho^{*}\left(Y+\varepsilon\left(X_{1}-Y_{1}\right)\right)-\rho^{*}(Y)}{\varepsilon}$

where $\rho^{*}(X):=\min _{Z_{1}+Z_{2}=X}\left(\rho_{1}\left(Z_{1}\right)+\rho_{2}\left(Z_{2}\right)\right)$.

Detail. In this case, the directional derivative in (30) exists due to continuity of $Y$.

\section{Conclusions}

We have reduced the problem of identifying the unique linear diversifying risk contribution $\Lambda_{\rho}(X, Y)$ to the problem of selecting the unique center of a convex weakly compact set in a Banach space. The latter problem has a natural solution (centroid) in finitedimensional case, which corresponds to the case when probability space $\Omega$ is finite. In the general case, we have used the construction of Lim (1981), which has the advantage that the resulting center is a fixed point of every affine isometry mapping the set into itself. This construction produces the unique risk contribution, defined for all $X, Y \in \mathcal{L}^{\infty}(\Omega)$, and for every coherent risk measure $\rho$, as well as for other classes of functionals, such as deviation measures or error measures. Our solution is consistent with those derived in Kalkbrener (2005) and Cherny and Orlov (2011) in cases the latter exist and unique. It can also be applied to selecting the unique Pareto optimal allocation in a wide class of optimal risk sharing problems.

Acknowledgments. The author would like to thank the University of Leicester for supporting this research in granting him academic study leave.

\section{References}

[1] Aase, K. (2002). Perspectives of risk sharing. Scandinavian Actuarial Journal, 2, 73-128.

[2] Arrow, K. J. (1963). Uncertainty and the welfare of medical care. American Economic Review, 53, 941-973.
[3] Artzner, P., Delbaen, F., Eber, J.-M., \& Heath, D. (1999). Coherent measures of risk. Mathematical Finance, 9, 203-227.

[4] Becker, R. (1991). Fundamental theorems of welfare economics in infinite dimensional commodity spaces. In: Equilibrium Theory in Infinite Dimensional Spaces, Ali Khan, M. and Yannelis N., ed. Studies in Economic Theory, 1, Springer, 124-175.

[5] Borch, K. H. (1962). Equilibrium in a reinsurance market. Econometrica, 30, 424-444.

[6] Brodskii, M. S., \& Mil'man, D. P. (1948). On the center of a convex set, Dokl. Akad. Nauk SSSR, 59, 837-840. MR 9,448.

[7] Burgert, C. \& Ruschendorf, L. (2008). Allocation of risks and equilibrium in markets with finitely many traders. Insurance: Mathematics and Economics, 42(1), 177-188.

[8] Cherny, A. S. (2006). Weighted VaR and its properties. Finance and Stochastics, 10(3), 367-393.

[9] Cherny, A. S., \& Orlov, D. (2011). On two approaches to coherent risk contribution, Mathematical Finance, 21(3), 557-571.

[10] Dana, R.-A. (2005). A representation result for concave Schur-concave functions. Mathematical Finance 15(4), 613-634.

[11] Dana, R.-A., \& Le Van, C. (2010). Overlapping sets of priors and the existence of efficient allocations and equilibria for risk measures. Mathematical Finance 20(3), 327-339.

[12] Delbaen, F. (2004). Coherent monetary utility functions, Working Paper, available at http://www.math.ethz.ch/ delbaen/ under the name "Pisa lecture notes."

[13] Denault, M. (2001). Coherent allocation of risk capital. Journal of Risk 4(1), 1-34.

[14] Filipovic D., \& Kupper M. (2008). Equilibrium Prices for monetary utility functions. International Journal of Theoretical and Applied Finance, 11, 325-343. 
[15] Föllmer, H., \& Schied, A. (2004). Stochastic finance, (2nd ed.). Berlin New York: de Gruyter.

[16] Grechuk, B., Molyboha, A., \& Zabarankin, M. (2013). Cooperative games with general deviation measures. Mathematical Finance, 23(2), 339-365.

[17] Grechuk, B., \& Zabarankin, M. (2012a). Optimal risk sharing with general deviation measures. $A n$ nals of Operations Research, 200(1), 9-21.

[18] Grechuk, B., \& Zabarankin, M. (2012b). Schur convex functionals: Fatou property and representation. Mathematical Finance, 22(2), 411-418.

[19] Heath, D., \& Ku, H. (2004). Pareto equilibria with coherent measures of risk. Mathematical Finance, 14, 163-172.

[20] Hunt, B. R., Sauer, T., \& Yorke, J. A. (1992). Prevalence: a translation-invariant "almost every" on infinite-dimensional spaces. Bulletin of the American Mathematical Society, 27(2), 217238.

[21] Jouini, E., Schachermayer, W., \& Touzi, N. (2006). Law invariant risk measures have the Fatou property. Advances in Mathematical Economics, 9, 49-71.

[22] Jouini, E., Schachermayer, W., \& Touzi, N. (2008). Optimal risk sharing for law-invariant monetary utility functions. Mathematical $\mathrm{Fi}$ nance, 18(2), 269-292.

[23] Kalkbrener, M. (2005). An axiomatic approach to capital allocation. Mathematical Finance, 15(3), 425-437.

[24] Lim, T. C. (1981). The center of a convex set. Proceedings of the AMS, 81(2), 345-346.

[25] Lesnevski, V., Nelson, B. L., \& Staum, J. C. (2007). Simulation of coherent risk measures based on generalized scenarios. Management Science, 53(11), 1756-1769.

[26] Rockafellar, R.T., Uryasev, S., \& Zabarankin, M. (2006a). Generalized deviations in risk analysis. Finance and Stochastics, 10(1), 51-74.
[27] Rockafellar, R.T., Uryasev, S., \& Zabarankin, M. (2006b). Optimality conditions in portfolio analysis with general deviation measures. Mathematical Programming, 108(2-3), 515-540.

[28] Rockafellar, R.T., Uryasev, S., \& Zabarankin, M. (2008). Risk tuning with generalized linear regression. Mathematics of Operations Research 33(3), 712-729.

[29] Rudloff, B. (2006). Hedging in incomplete markets and testing compound hypotheses via convex duality. Dissertation. Martin-Luther University Halle-Wittenberg. 